J3eA, Journal sur l'enseignement des sciences et technologies de l'information et des systèmes, Volume 3, Hors-Série 1, 7 (2004)

DOI : http://dx.doi.org/10.1051/bib-j3ea:2004607

(C) EDP Sciences, 2004

\title{
Perception par ultrasons et vision pour la localisation d'un fauteuil électrique autonome
}

O. Horn et M. Kreutner

Laboratoire d'Automatique des Systèmes Coopératifs (L.A.S.C.)

Île du Saulcy

BP 80794

F-57012 Metz CEDEX 01, France

\{horn, kreutner\}@lasc.sciences.univ-metz.fr 


\title{
Perception par Ultrasons et Vision pour la Localisation d'un Fauteuil Electrique Autonome
}

\author{
O. Horn, M. Kreutner \\ Laboratoire d'Automatique des Systèmes Coopératifs (L.A.S.C.) \\ Ile du Saulcy, BP 80794, 57012 METZ Cedex 01 France \\ \{horn, kreutner\}@lasc.sciences.univ-metz.fr
}

Mots clefs : fauteuil électrique, capteurs ultrasons, vision, localisation.

\section{INTRODUCTION}

Cet article traite du mode d'analyse des données issues de capteurs odométriques ultrasonores et de vision pour déterminer la position d'un fauteuil électrique autonome. Ce dernier est conçu pour donner une mobilité aux personnes dont les handicaps moteurs interdisent la conduite des fauteuils électriques classiques.

\section{A. Contexte du projet}

Pour un certains nombre de personnes atteintes de forts handicaps moteurs le besoin d'assistance à la mobilité ne peut être assuré par des fauteuils électriques classiques. En effet en raison de possibilités physiques trop faibles ou d'incapacités cognitives il leur est impossible de manipuler correctement un joystick. En revanche une commande tout ou rien constitue un mode de communication accessible. C'est pourquoi les récentes avancées technologiques et méthodologiques en robotique mobile ont pu s'ouvrir à un nouveau champ d'application : les véhicules autonomes ou semi-autonomes pour handicapés moteur. Leur objectif est de rendre les déplacements possibles grâce à un fauteuil « intelligent» qui allège les taches de conduite. Pour ce faire un ordinateur est embarqué sur le fauteuil et commande différents modules afin de réaliser le trajet choisi par la personne. Plusieurs études ont été menées pour définir et réaliser ce type de dispositif. Certaines ont consisté à établir des primitives réactives qui permettent d'ajuster les trajectoires par rapport à l'environnement proche perçu par les capteurs extérioceptifs[1] [2]. D'autres visent à confier la maîtrise du mouvement à l'ordinateur [3] [4] ce qui nécessite l'établissement de primitives de haut niveau comme la planification et le suivi de trajectoire.

\section{B. Contraintes et possibilités}

Le projet VAHM [5] que nous développons actuellement au LASC, vise à mettre en place un fauteuil équipé qui adapte au mieux la répartition des tâches entre l'homme et la machine, suivant le degré de handicap de l'utilisateur et la complexité de l'environnement. A cet effet nous avons défini différents modes de fonctionnement, pour lesquels le rôle du système varie d'un soutien partiel à partir de primitives locales jusqu'à une prise en charge totale du mouvement pour lequel la personne indique seulement le but à atteindre. Ce dernier mode de fonctionnement (automatique) est défini dans un espace connu et modélisé et nécessite notamment que le fauteuil soit en mesure de se situer dans son environnement.

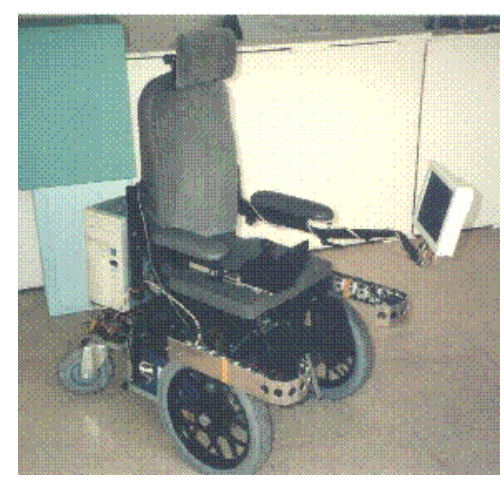

Fig.1. Le prototype du V.A.H.M.

Le prototype du robot VAHM est actuellement implanté sur un fauteuil électrique Power Push ${ }^{\mathrm{TM}}$ (Figure 1) équipé de 16 capteurs ultrasons Polaroïd ${ }^{\mathrm{TM}}$, de codeurs incrémentaux (un sur chaque roues motrices) et d'un microordinateur. Le système de communication hommemachine est constitué d'un écran où la machine affiche ses informations et requêtes et d'un interrupteur permettant à l'utilisateur de donner ses instructions.

Lorsque la personne demande au fauteuil d'atteindre un objectif dans un univers modélisé, il est nécessaire, pour que le déplacement s'exécute correctement, que le fauteuil soit localisé au départ afin de planifier son mouvement à partir de ce point, puis que la position soit contrôlée en cours de mouvement pour s'assurer que la trajectoire est correctement suivie. Deux procédures distinctes sont nécessaires car le contexte et les contraintes sont différents dans les deux cas (figure 2):

- à l'arrêt, les seules informations disponibles sont les mesures ultrasonores et une indication de l'utilisateur concernant la zone dans laquelle il se trouve. Le temps de traitement peut être de l'ordre de la minute et la précision requise est de l'ordre de $10 \mathrm{~cm}$ en position et $7^{\circ}$ en orientation. En effet cet ordre de grandeur est acceptable pour établir la planification du déplacement et pour initialiser la position odométrique qui sera ajustée par la seconde procédure.

- $\quad$ en cours de mouvement la localisation consiste en un recalage de la position odométrique grâce aux mesures ultrasonores et de vision pour assurer une exécution correcte de la trajectoire le temps de traitement autorisé est 
de quelques secondes et la précision requise est de l'ordre de $5 \mathrm{~cm}$ en position et $3^{\circ}$ en orientation.

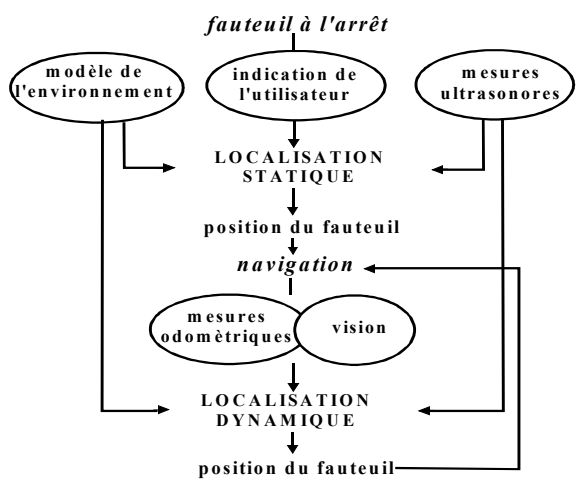

Fig. 2. Deux modes de localisation du V.A.H.M.

Nous décrivons dans cet article le mode d'enregistrement et d'interprétation des mesures et leur emploi pour réaliser la localisation statique et la localisation dynamique, dans l'environnement connu du fauteuil.

\section{LA LOCALISATION STATIQUE}

Nous traitons ici de la localisation statique du fauteuil qui intervient pour l'initialisation de la position odométrique ou, après un déplacement, si une erreur odométrique trop grande n'a pas permis d'exploiter la localisation dynamique tout au long de la trajectoire. Dans ce contexte, il existe une multitude de méthodes de localisation différentes suivant l'application et les capteurs utilisés ; par exemple en utilisant des capteurs à ultrasons $[6][7][8]$, un système de vision [9][10] ou encore en alliant la vision à un laser [11].

\section{A. Principe}

Dans notre cas, les seules informations disponibles sont : une connaissance précise de l'environnement (plan d'architecte, emplacement des meubles), les mesures des capteurs à ultrasons et une information vague sur la position $\mathrm{du}$ robot. Cette dernière information provient soit de l'utilisateur au démarrage, soit du module de navigation quand l'erreur odométrique sur la position devient trop grande. Nous disposons de peu de mesures et d'une connaissance précise de l'environnement. Nous avons adopté la structure de grille qui permet la représentation la plus fine des mesures. Nous définissons deux grilles : la grille locale qui représente les mesures et la grille globale qui représente l'environnement connu d'évolution du robot.

La grille globale est définie à partir du plan de l'environnement et de la place des meubles, et enregistrée sous forme de cellules affectées à des valeurs positives pour représenter les murs et les objets et à des valeurs négatives pour représenter l'espace libre. La grille locale, décrit les limites de l'espace libre autour du robot grâce aux données des capteurs à ultrasons. Pour une mesure donnée la région correspondant aux positions possibles de l'obstacle détecté est un arc centré sur le capteur dont les cellules prendront des valeurs positives. Et le secteur compris entre cet arc et le capteur constitue l'espace libre perçu représenté par des cellules à valeur négatives. La grille locale est centrée sur l'axe de rotation du robot et l'objectif de la localisation est de déterminer la position et l'orientation du robot dans son environnement. Nous recherchons donc la position de meilleure correspondance entre la grille locale et la grille globale.

Cette définition de la méthode de localisation est similaire aux méthodes employées pour l'évitement d'obstacle[12] ou la construction de carte simultanée à la localisation[13]. Cependant notre problématique est différente dans la mesure où nous disposons d'un environnement précisément modélisé et notre objectif est de déterminer la place exacte $d u$ fauteuil dans cet environnement. Le fauteuil étant à l'arrêt la contrainte de temps n'est pas déterminante mais il nous est impossible de rechercher des mesures complémentaires nécessitant un déplacement $\mathrm{du}$ fauteuil qui gènerait la personne. Notre travail s'est donc focalisé sur l'interprétation la plus fine possible de chacune des connaissances dont nous disposons pour localiser le robot.

\section{B. Représentation des données et de l'environnement}

Dans le modèle local des mesures de grandes distance peuvent coexister avec des mesures plus petites. Or une grande mesure couvrant plus de cellules qu'une petite sa contribution risque d'être plus importante alors qu'elle est plus susceptible d'être bruitée. Pour équilibrer l'influence de chaque mesure et donner le même poids à chaque capteur, nous considérons le nombre de cellules couvertes par une zone (vide ou occupée) et affectons à chacune de ses cellules une valeur inversement proportionnelle à ce nombre. Par conséquent, pour chaque cône représentant une mesure ultrasonore, nous donnons aux $\mathrm{n}_{1}$ cellules de l'arc la valeur $1 / \mathrm{n}_{1}$ et aux $\mathrm{n}_{2}$ cellules du secteur la valeur $-1 / \mathrm{n}_{2}$, (figure 3).

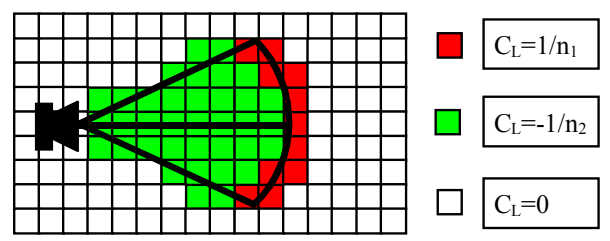

Fig.3. Enregistrement de mesure du capteur ultrason.

Par ailleurs nous avons traité le cas de mesures dont les cônes d'émissions se superposent et délivrent des valeurs contradictoires pour les cellules. Pour cela nous avons considéré les remarques d'autres chercheurs ayant travaillé sur les capteurs ultrasons qui établissent une bonne fiabilité de ces derniers concernant la détection des espaces libres et une incertitude conséquente pour le lieu des obstacles. 


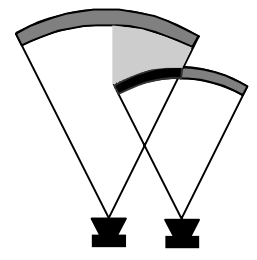

Mesures contradictoires

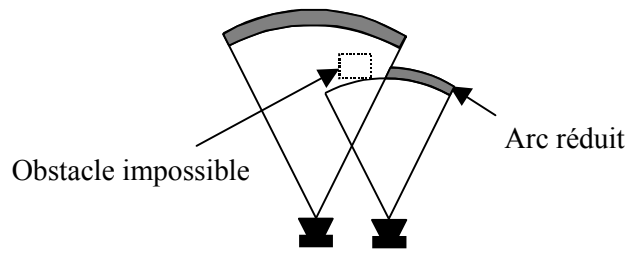

interprétation

Fig.4. Interprétation de mesures contradictoires.

Nous en concluons que lors de mesures contradictoires l'espace vide est prioritaire sur l'espace occupé. Nous écrasons donc la partie de l'arc occupé qui se situe sur la zone vide d'un autre capteur. Compte tenu de cela l'arc occupé de la petite mesure est réduit et la valeur des cellules le composant est ajustée (Figure 4).

Nous considérons aussi les cellules couvertes par la surface du robot, sachant qu'elles représentent nécessairement un espace libre et afin de leur donner la même contribution que l'espace libre défini par les capteur elles prennent la valeur $-\mathrm{c} / \mathrm{n}$ où $\mathrm{c}$ est le nombre de capteur et $\mathrm{n}$ le nombre de cellules considérées.

Dans un premier temps l'environnement de travail avait été enregistré sous forme de cellules prenant la valeur -1 pour l'espace libre et 1 pour les parties occupées (mur ou meubles). Les premiers essais ont montré que ce modèle naturel peut mettre en défaut notre mode de localisation. En effet l'arc occupé du capteur tendait à se positionner à l'intérieur de l'obstacle alors qu'en toute rigueur cet arc doit être tangent à la limite de l'obstacle et de ce fait la position délivrée par l'algorithme pouvait être décalée par rapport à la position réelle.

Pour éviter ce type d'erreur nous considérons le modèle global non plus comme une représentation de l'environnement dans sa réalité mais dans la perception que peuvent en avoir les capteurs U.S.. Ces derniers n'observent pas tout l'espace occupé mais seulement la limite entre espace libre et espace occupé sur laquelle l'onde se réfléchit. De leur point de vue seules les cellules situées à la limite des obstacles doivent être enregistrées avec une valeur positive les cellules représentant l'intérieur des obstacles doivent être considérées comme inconnues. Nous modifions donc la grille globale en ne maintenant la valeur 1 que pour les cellules situées à la frontière des obstacles les autres cellules sont mises à 0 (Figure 5).
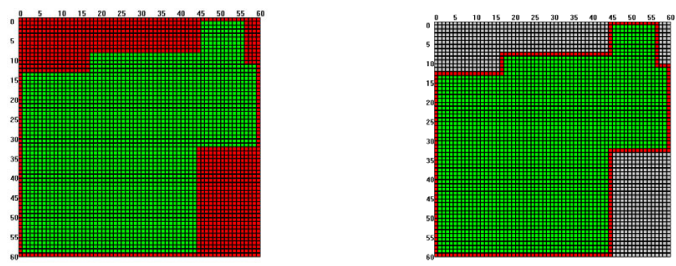

Fig.5. Modification de la grille globale.

\section{Implantation et résultats}

L'objectif de la localisation est de déterminer la position et l'orientation du robot dans son environnement. Nous recherchons donc la position de meilleure correspondance entre la grille locale et la grille globale. Partant d'une position et orientation données, nous recherchons la translation $(a, b)$ et la rotation ( ) qui feront coïncider au mieux les deux grilles.

Ce calcul est réalisé en recherchant le maximum du critère de correspondance $S(a, b, \theta)$ défini par :

$$
\left.S(a, b, \theta)=\sum_{i, j \in \text { grilllbcale }}\left(\mathrm{C}_{\mathrm{L}}(i, j) \cdot \mathrm{C}_{\mathrm{G}}(k, l)+\mid \mathrm{C}_{\mathrm{L}}(i, j)\right) \cdot\left(\mathrm{C}_{\mathrm{G}}(k, l)-1\right)\right)
$$

Avec $C_{L}$ valeurs des cellules de la grille locale $\mathrm{C}_{\mathrm{G}}$ valeurs des cellules de la grille globale

$$
\left\{\begin{array}{l}
k=a+\left(i-i_{0}\right) \cdot \cos \theta+\left(j-j_{0}\right) \cdot \sin \theta \\
1=b-\left(i-i_{0}\right) \cdot \sin \theta+\left(j-j_{0}\right) \cdot \cos \theta
\end{array}\right.
$$

$\mathrm{i}_{0}$ et $\mathrm{j}_{0}$ sont les indices de la cellule de la grille locale représentant l'axe de rotation du robot.

Le terme $\left|C_{L}(i, j)\right| \cdot\left(\left|C_{G}(k, 1)-1\right|\right)$ est nul si $C_{L}=0$ ou si $\mathrm{C}_{\mathrm{G}} \neq 0$ mais permet de décrémenter $\mathrm{S}$ lorsque $\mathrm{C}_{\mathrm{L}} \neq 0$ et $\mathrm{C}_{\mathrm{G}}=0$ c'est à dire lorsque le cône d'émission d'un capteur mord sur un obstacle.

Nous avons implanté l'algorithme sur le prototype $\mathrm{du}$ V.A.H.M. que nous testons dans un appartement reconstitué au laboratoire composé de plusieurs pièces dans lequel le robot se déplace. Nous avons lancé la procédure de localisation en une centaine d'emplacements différents. Pour évaluer la qualité des résultats nous avons mesuré manuellement la place exacte du robot pour chaque test.

Pour ces essais, la localisation du robot est réalisée deux fois : une première fois en ligne sur le robot avec les mesures des capteurs à ultrasons puis une seconde à partir de mesures simulées. Afin de nous placer dans les conditions réelles d'utilisation de la méthode, nous fournissons pour chaque essais une évaluation imprécise de la place du robot qui est obtenue en estimant visuellement sa position et son orientation. La recherche est réalisée sur une surface de $\pm 75 \mathrm{~cm}$ autour de cette position estimée et pour des orientations comprises dans un cône de $\pm 45^{\circ}$ autour de l'orientation estimée. La 

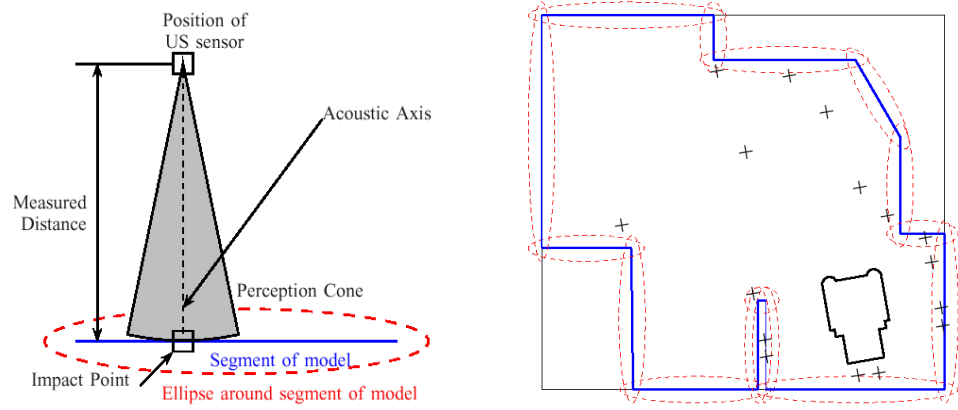

Fig.7. Modélisation du capteur ultrason et projection de la carte locale sur la carte globale.

résolution des grilles employées est de 10x10 $\mathrm{cm}$ par cellule. Le pas de recherche est d'une cellule pour la position et de 5 degrés pour l'orientation. Sachant qu'une bonne localisation admet une erreur maximale en position d'une cellule $( \pm 10 \mathrm{~cm})$ et une différence maximale en orientation de $7^{\circ}$, à partir de 100 essais réalisés nous obtenons un taux de réussite de $80 \%$ avec les valeurs des capteurs mesurées et de $100 \%$ avec les valeurs des capteurs simulées.

Quelques exemples sont donnés sur la figure cidessous (Figure 6), la position réelle est représentée en noir la position calculée hors ligne par les mesures simulées en gris et la position calculée sur le robot par les mesures des capteurs en blanc.

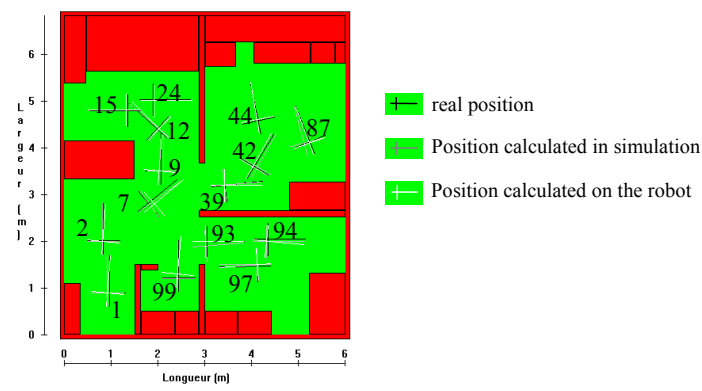

Fig.6. Exemples d'essais réalisés dans l'appartement de test.

\section{LA LOCALISATION DYNAMIQUE}

Il s'agit ici d'utiliser les mesures ultrasonores comme information complémentaire des mesures odométriques pour déterminer la position du fauteuil. Pour cela différentes méthodes existent qui diffèrent par le mode d'association choisi entre les mesures. Le filtre de Kalman est souvent utilisé [14][15] en prenant la position odomètrique comme valeur prédite et les mesures ultrasonores comme innovation ou le critère des moindres carrés peut être employé pour associer les mesures et l'environnement en utilisant la position odomètrique comme point de départ [16].

\section{A. Exploitation des mesures ultrasonores}

Dans notre contexte le réajustement se fait en cours de mouvement environ tous les $50 \mathrm{~cm}$, il doit donc traiter de faibles dérives et s'exécuter instantanément. La méthode des moindres carrés permet de rechercher rapidement la transformation à apporter à la position odométrique pour obtenir la meilleure association entre les mesures et l'environnement. Partant de ce principe nous réalisons la localisation dynamique en construisant une carte ponctuelle locale des obstacles perçus par les capteurs ultrasons autour du robot puis en recherchant le petit ajustement de la position odomètrique qui associera au mieux (selon le critère des moindres carrés) cette carte ponctuelle avec la carte globale de l'environnement.

Pour la carte locale chaque obstacle perçu est représenté par un point dans l'axe acoustique du capteur. Puis la carte locale est projetée sur la carte globale autour de la position estimée du robot (figure 7), cette dernière est initialisée par la position odométrique puis ajustée à chaque itération de l'algorithme. Pour chaque point le segment dont il est le plus proche est défini par l'ellipse le recouvrant. Certains points ne seront affectés à aucun segment. La méthode des moindres carrés calcule à partir des points retenus la petite transformation (translation et rotation) qui minimisera la somme des distances pointsdroites au carré. A partir de cette transformation la position estimée est ajustée et la procédure relancée jusqu'à obtenir une transformation de faible amplitude (inférieure à $5 \mathrm{~cm}$ en translation et à $5^{\circ}$ en orientation).

\section{B. Essais sur le VAHM}

Nous avons mis en œuvre cette méthode sur le prototype du VAHM. Nous avons placé le véhicule en différentes positions dans un environnement et relevé sa position au sol pour chaque acquisition. L'algorithme de correction est initialisé avec une position estimée comprise dans une région de $20 \mathrm{~cm}$ de côté centrée autour de la position relevée au sol et avec une orientation estimée comprise dans un intervalle de $\pm 10^{\circ}$ autour de l'orientation relevée au sol, tout en évitant les initialisations qui nous placeraient dans la zone où les erreurs en position et orientation sont inférieures à leurs conditions d'arrêt respectives. 
Sur l'ensemble des essais réalisés nous observons une réduction de l'erreur moyenne en position, de $10.7 \mathrm{~cm}$ à $7.4 \mathrm{~cm}$ et la fréquence d'apparition d'erreurs importantes après correction est faible [17]. En revanche, l'algorithme n'obtient pas d'aussi bons résultats pour la correction en orientation. En effet l'erreur moyenne (environ $7^{\circ}$ ) reste quasiment la même après correction.

$\mathrm{Ce}$ problème est principalement dû aux caractéristiques des capteurs ultrasons, en effet nous considérons que la mesure se situe sur l'axe de tir du capteur alors que la zone de perception est un cône autour de cet axe. Il parait impossible d'ajuster l'orientation au moyen de capteurs ultrasons fixes, cela induit la nécessité d'employer un autre type de capteur: un capteur de vision. La partie suivante va traiter de la méthode d'ajustement de l'orientation par vision monoculaire.

\section{Ajustement de l'orientation par vision monoculaire}

Le système de vision est composé d'une caméra CCD fixée sur l'un des accoudoirs du fauteuil de sorte que son axe optique soit orienté dans la direction du mouvement $\mathrm{du}$ fauteuil. L'algorithme utilise l'image acquise par la caméra dont il extrait certaines caractéristiques qu'il identifie dans le modèle de l'environnement. Connaissant la position estimée du robot, l'orientation est ajustée en mesurant la place dans l'image des repères connu de l'environnement.

Les composantes utilisés sont les verticales que l'on peut observer dans un environnement intérieur (montant de porte, poster, pied de table...) en effet ce type de caractéristique employée avec une caméra permettent des mesures d'orientation précises [18]. Le mode d'interprétation des données consiste à calculer l'orientation de la caméra à partir de la représentation d'une caractéristique dans l'image (Figure 8).

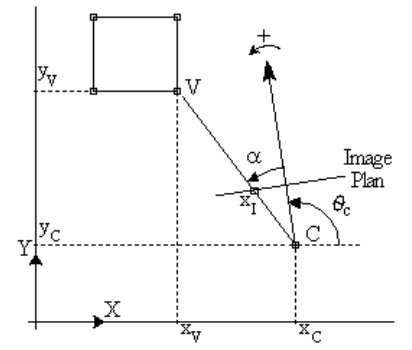

Fig.8. Paramètres employés pour le calcul de l'orientation.

Connaissant la position de la caméra $\left(x_{C}, y_{C}\right)$, la position d'un repère vertical donné dans le modèle $\left(x_{V}, y_{V}\right)$ et $x_{I}$ l'abscisse du contour correspondant vu dans le plan image, l'orientation de la caméra est déterminée par la relation suivante:

$$
\theta_{C}=\arctan \left(\frac{y_{V}-y_{C}}{x_{V}-x_{C}}\right)-\alpha \quad \text { avec } \alpha=\frac{x_{I}}{f}
$$

où $\alpha$ est l'angle sous lequel cette verticale est vue par la caméra et $f$ la distance focale de la caméra.

Le problème de détermination de l'orientation devient alors la recherche de correspondance entre segments verticaux de l'environnement et contours détectés dans l'image. A cette fin nous définissons un arbre d'interprétation où chaque niveau représente un contour et chaque nœud une mise en correspondance d'un segment avec ce contour.

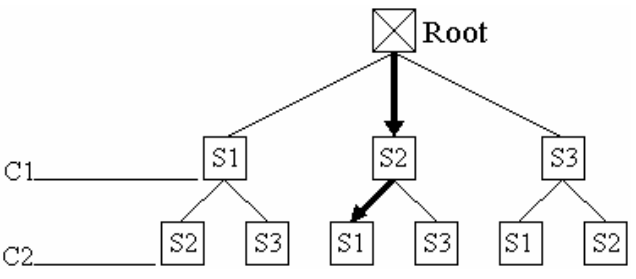

Fig.9. Exemple d'arbre d'interprétation avec 2 contours et 3 segments.

A partir des équations ci dessus, chaque nœud représente une orientation de la caméra et le chemin qui représente la bonne mise en correspondance entre contours et segments est celui pour lequel les orientations successives sont sensiblement les mêmes.

La correction de l'orientation sera réalisée de la manière suivante:

- déterminer les segments visibles de la position estimée du fauteuil à partir du modèle de l'environnement.

- extraire les contours verticaux de l'image perçue par la caméra (filtrage de Deriche)

- mettre en correspondance contours et segments (arbre) et en déduire l'orientation de la caméra et donc du fauteuil.

Nous avons mis en œuvre cette méthode sur le prototype du VAHM [17]. L'algorithme de correction est initialisé avec une position estimée égale à la position relevée au sol avec différentes orientations estimées, jusqu'à ce que l'orientation corrigée ne satisfasse plus aux conditions requises (erreur $<3^{\circ}$ ).

Le tableau suivant donne les plages de variations de l'erreur d'estimation initiale admissible.

\begin{tabular}{|c|c|c|}
\hline Cas & $\begin{array}{l}\text { Estimation } \\
\text { Error Range }\end{array}$ & $\begin{array}{l}\text { Error After } \\
\text { Adjustment }\end{array}$ \\
\hline 00 & $-13^{\circ}$ to $12^{\circ}$ & $<1^{\circ}$ \\
\hline 01 & - & $>5^{\circ}$ \\
\hline 02 & $-13^{\circ}$ to $17^{\circ}$ & $<1^{\circ}$ \\
\hline 03 & $-10^{\circ}$ to $23^{\circ}$ & $<3^{\circ}$ \\
\hline 04 & $-13^{\circ}$ to $14^{\circ}$ & $<2^{\circ}$ \\
\hline 06 & $-12^{\circ}$ to $10^{\circ}$ & $<1^{\circ}$ \\
\hline 07 & $-11^{\circ}$ to $10^{\circ}$ & $<2^{\circ}$ \\
\hline 08 & $-14^{\circ}$ to $14^{\circ}$ & $<1^{\circ}$ \\
\hline 09 & $-12^{\circ}$ to $13^{\circ}$ & $<3^{\circ}$ \\
\hline 10 & $-10^{\circ}$ to $18^{\circ}$ & $<2^{\circ}$ \\
\hline $11-12$ & - & - \\
\hline 13 & $-11^{\circ}$ to $10^{\circ}$ & $<1^{\circ}$ \\
\hline 14 & $-13^{\circ}$ to $12^{\circ}$ & $<3^{\circ}$ \\
\hline 15 & $-16^{\circ}$ to $12^{\circ}$ & $<1^{\circ}$ \\
\hline 16 & $-10^{\circ}$ to $16^{\circ}$ & $<1^{\circ}$ \\
\hline
\end{tabular}


Excepté les cas (11) et (12) pour lesquels une seule verticale est présente dans l'image rendant l'algorithme inopérant, les résultats obtenus avec la méthode d'ajustement de l'orientation satisfont aux objectifs fixés lorsqu'il n'y a pas de contours parasites dans l'image. Dans le cas (01) la présence d'un tel contour rend la correction impossible, mais si on élimine ce contours non représentatif d'une caractéristique de l'environnement, l'ajustement donne le bon résultat.

Notre travail consiste actuellement à développer une version de l'algorithme qui permet d'annuler l'effet des contours parasites. Pour cela une verticale fictive est introduite dans le modèle qui sera associée au contour parasite pour créer un "nœud vide", ce qui permet de ne plus considérer ce contour dans l'arbre d'interprétation pour le calcul de l'orientation.

\section{CONCLUSION}

Les travaux décrits dans cette communication visent à établir le mode de localisation d'un fauteuil électrique autonome qui satisfasse aux contraintes suivantes:

. pouvoir être employé dans tout type d'environnement sans équipement particulier

- employer les mesures issues des capteurs équipant le fauteuil (odométrie, ultrasons, vision)

. ne pas nécessiter de déplacements destinés à obtenir des mesures complémentaires

- donner des résultats suffisamment précis pour planifier et exécuter une trajectoire.

Nous avons établis deux approches différentes selon le contexte de leur emploi: à l'arrêt et en cours de mouvement. La méthode statique repose sur une représentation précise des mesures ultrasonores et permet de définir une position du fauteuil suffisamment précise pour établir sa trajectoire. La méthode dynamique (plus rapide) consiste en un ajustement de la position odométrique à partir d'une interprétation grossière des mesures ultrasonores, puis en un ajustement de l'orientation issue de cette première procédure au moyen d'une caméra. Les résultats obtenus lors des tests réalisés en condition réelles montrent l'adéquation des méthodes à notre projet.

\section{RÉFÉRENCES}

[1] LEVINE S.P., BELL D.A., JAROS L.A., SIMPSON R.C., KOREN Y.K., BORENSTEIN J., "The navchair assistive wheelchair navigation system", IEEE Transactions on Rehabilitation Engineering, vol. 7, $\mathrm{n}^{\circ} 4$, pp. 443-451, 1999.

[2] PRASSLER E., SCHOLZ J., FIORINI P., "A Robotic Wheelchair for Crowded Public Environements", IEEE Robotics and Automation Magazine Vol.7, $\mathrm{N}^{\circ} 1$, March 2001, pp. 38-45.

[3] BORGOLTE U., HOYER H., BUHLER C., HECK H., HOEPLER R., "Architectural concepts of a semi- autonomous wheelchair" , Journal of Intelligent and Robotic Systems 22, pp. 233-253, 1998.

[4] YODER J.D., BAUMGARTNER E.T., SKAAR S.B., "Initial results in the development of a guidance system for a powered wheelchair" IEEE Transactions on Rehabilitation Engineering, Vol 4, n³, pp. 143-151, 1996.

[5] G. BOURHIS, O. HORN, O. HABERT, A. PRUSKI, «The VAHM Project: Autonomous Vehicle for People with Motor Disabilities » IEEE Robotics and Automation Magazine Vol.7, N¹, March 2001, pp. 21-28.

[6] DRUMHELLER M., "Mobile Robot Localization Using Sonar", IEEE Transactions on Pattern Analysis and Machine Intelligence, Vol 9, $\mathrm{N}^{\circ}$ 2, March 1987, pp 325-332.

[7] CROWLEY J.L.: "World modeling and position estimation for a mobile robot using ultrasonic ranging", Proceedings of the IEEE International Conference on Robotics and Automation, 1989.

[8] BEOM H.R., CHO H.S. : " Mobile robot localization using a single rotating sonar and two passive cylindrical beacons ", Robotica, vol 13, 1995, pp. 243-252.

[9] MADSEN C.B., ANDERSEN C.S.: " Optimal landmark selection for triangulation of robot position", Robotics and Autonomous Systems, vol 23, 1998, pp. 277-292.

[10] MARHIC B., MOUADIB E.M., PEGARDC., HUTTIN N. : " Localisation Based on Invariant-Models Recognition by SYCLOP", Proceedings of the IEEE/RSJ International Conference on Intelligent Robots and Systems, IROS'99, 1999, pp. 793-798.

[11] ARRAS K.O., TOMATIS N. : " Improving Robustness and Precision in Mobile Robot Localization by Using Laser Range Finding and Monocular Vision", $3^{\text {rd }}$ European Workshop on Advanced Mobile Robots (Eurobot'99), Zurich, Switzerland, september 6-8, 1999.

[12] LANKENAU A., ROFER T.: "A Versatile and Safe Mobility Assistant" IEEE Robotics and Automation Magazine, Vol.7, n²1, 2001, pp.29-37.

[13] SCHULTZ A.C., ADAMS W., YAMAUCHI B., "Integrating exploration, localization, navigation, and planning with a common representation", Autonomous Robots 6, pp. 293-308,1999.

[14] LEONARD J.J., DURRANT-WHYTE H.F., "Mobile Robot Localization by Tracking Geometric Beacons", IEEE Transactions on Robotics and Automation, Vol. 7, n'3, pp. 376-382, 1991.

[15] ARSENIO A., RIBEIRO I., "Active range sensing for mobile robot localization", IROS'98, Victoria, Canada, pp. 1066-1071,1998.

[16] COX I.J., "Blanche- An Experiment in Guidance and Navigation of an Autonomous Robot Vehicle ", IEEE Transactions on Robotics and Automation, vol. 7, $\mathrm{n}^{\circ} 2$, 1991, pp. 193-204.

[17] KREUTNER M., HORN O., " Co-operation between ultrasound and monocular vision for the localization of a mobile robot", multiconférence 'Traitement de l'Information dans la Théorie des Systèmes et ses 
Applications' (CESA'2003), 07/2003 Lille, France (à paraître).

[18] MADSEN C., ANDERSEN C., "Optimal Landmark Selection for Triangulation of Robot Position" IEEE Robotics and Autonomous Systems, vol. 23, n 4, 1998, pp.277-292. 